\title{
Comparing precipitation and temperature trends between inland and coastal locations
}

\author{
$\begin{array}{lll}\text { J. Hartigan } & \text { S. MacNamara } & \text { L. M. } \text { Leslie }^{3}\end{array}$
}

(Received 29 January 2019; revised 28 May 2019)

\begin{abstract}
Motivated by the Millennium Drought and the current drought over much of southern and eastern Australia, this detailed statistical study compares trends in annual wet season precipitation and temperature between a coastal site (Newcastle) and an inland site (Scone). Bootstrap permutation tests reveal Scone precipitation has decreased significantly over the past 40 years $(p$-value $=0.070)$ whereas Newcastle has recorded little to no change $(p$-value $=0.800)$. Mean maximum and minimum temperatures for Newcastle have increased over the past 40 years (p-values of 0.002 and 0.015 , respectively) while the mean maximum temperature for Scone has increased $(p$-value $=0.058)$ and the mean minimum temperature has remained stable. This suggests mean temperatures during the wet season for both locations are increasing. Considering these trends along with those for precipitation, water
\end{abstract}

DOI:10.21914/anziamj.v60i0.13967 gives this article, (c) Austral. Mathematical Soc. 2019. Published July 17, 2019, as part of the Proceedings of the 18th Biennial Computational Techniques and Applications Conference. ISSN 1445-8810. (Print two pages per sheet of paper.) Copies of this article must not be made otherwise available on the internet; instead link directly to the DOI for this article. 
resources in the Hunter region will be increasingly strained as a result of increased evaporation with either similar or less precipitation falling in the region. Wavelet analysis reveals that both sites have similar power spectra for precipitation and mean maximum temperature with a statistically significant signal in the two to seven year period, typically indicative of the El-Niño Southern Oscillation climate driver. The El-Niño Southern Oscillation also drives the Newcastle mean minimum temperature, whereas the Scone power spectra has no indication of a definitive driver for mean minimum temperature.

\section{Contents}

1 Introduction

C110

2 Data and methodology

C111

3 Results

C115

3.1 Evolution of precipitation . . . . . . . . . . .

3.2 Evolution of temperature . . . . . . . . . . . . C117

3.3 Wavelet analysis of precipitation . . . . . . . . . C C120

3.4 Wavelet analysis of temperature . . . . . . . . . C120

4 Discussion and Conclusions

\section{Introduction}

The onset of the current drought [2] was extremely sudden and affected much of southern and eastern Australia. This drought began in 2012, soon after the record breaking Millennium Drought of 1997-2009 which affected much of southern Australia and lasted well over ten years. In recent history a number of droughts have affected similar latitudes globally, including the 
Cape Town and California droughts $[6,5]$. These areas have observed an increase in drought frequency over the past few decades, consequent on the global warming trend. With changing precipitation patterns affecting areas of similar latitudes in southeast Australia, it is desirable to determine regions threatened by reduced annual wet season rainfall.

Mean temperatures over Australia have been increasing, particularly since the 1970s, and have been projected to continue increasing into the future [1]. An increase in temperature increases evaporation rates which can seriously affect water security. A decrease in precipitation in a region, combined with an increase in mean temperature and population, will tighten water availability, which can have vast economic and social impacts including decreased sanitation, enhanced fire risk, decreased irrigation affecting the agricultural sector and human health impacts. Few studies have assessed trends in precipitation within the Hunter region. This region contains both the second largest city in New South Wales, and a range of agricultural businesses. It has also been heavily affected by the current drought (Figure 1). This statistical study compares trends in annual wet season precipitation and temperature between a coastal site (Newcastle) and an inland site (Scone), which are both located within the Hunter region. An understanding of these trends is necessary for water resourcing efforts. Advanced statistical techniques are utilised to gain a deeper understanding of how precipitation and temperature have changed over time.

\section{Data and methodology}

Monthly mean maximum temperature (TMax), mean minimum temperature (TMin) and total monthly precipitation time series were obtained for Newcastle $\left(32.9^{\circ} \mathrm{S}, 151.8^{\circ} \mathrm{E}\right)$ and Scone $\left(32.1^{\circ} \mathrm{S}, 150.9^{\circ} \mathrm{E}\right)$ (Figure 1$)$ from the Climate Data Online section of the Australian Bureau of Meteorology (BoM) 


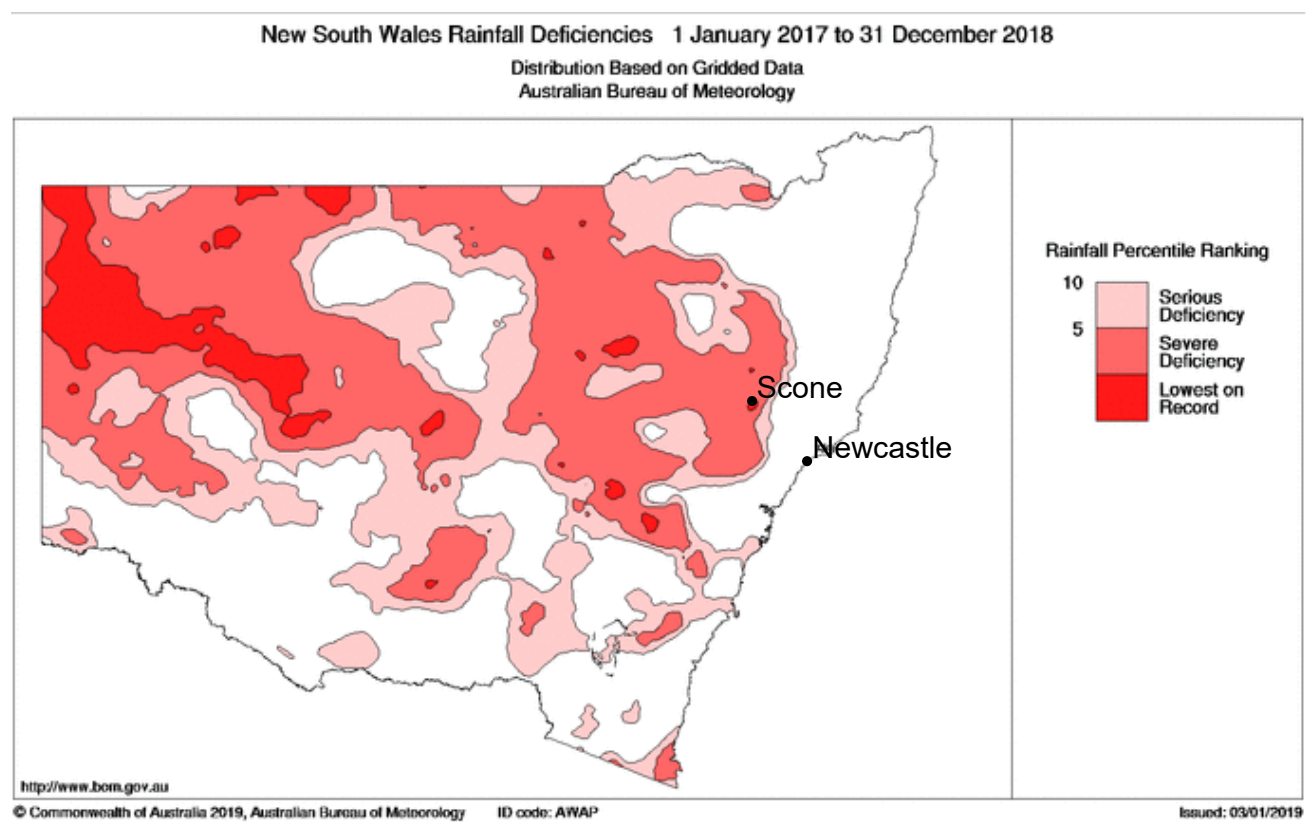

Product Code: IDCKAR75D0

Figure 1: Drought in NSW over the past 24 months, taken from the Bureau of Meteorology [3]. Locations of Newcastle and Scone are included.

website $^{1}$. Time series data were chosen from stations with relatively long records and few data gaps compared to other available weather station data within the one area. Nobby's Signal Station was used for Newcastle, which has a continuous record from the late 1800s. Meanwhile, multiple close sites within the township of Scone were utilised to create a long term data set. From 1957-1964 there were no temperature records made at Scone. To combat this, data from the nearby town of Jerrys Plains $\left(32.5^{\circ} \mathrm{S}, 150.9^{\circ} \mathrm{E}\right)$ was used with adjustments made based on the difference in mean temperature between the two sites. Any other missing data points were filled using the moving

${ }^{1}$ Australian Bureau of Meteorology http://www.bom.gov.au/climate/data/index. shtml 
average centred on the year for which the data was missing. The precipitation time series start at 1890 as this was the earliest year that both locations recorded precipitation, while the temperature time series start at 1910 as this is when consistent temperature recording practices were introduced in Australia.

The focus for this study is changes in precipitation and temperature for both locations during their respective wet seasons. To determine the wet season, monthly averages of precipitation over the entire time series were calculated and compared. Newcastle's wet season was found to run from January through to July, while Scone's wet season runs from October through to March. The time series were first graphed with percentiles to gain an overview of any general changes to precipitation or temperature. The data was then grouped into 20-year blocks and bootstrap resampling was applied with 5000 resamples to gain a deeper picture of any trends within the data. To test if there is a statistically significant difference in the mean of the distribution underlying two 20-year periods, permutation testing was applied, which is a resampling technique that does not require prior knowledge of the distribution.

Wavelet analysis was performed for both sites following the approach of Torrence and Compo [7] and using the accompanying MATLAB software. This computational technique is well suited to our data as wavelets provide information on the time evolution of the periodicity of signals within a time series, which is useful for detecting potential climate drivers such as the El-Niño Southern Oscillation (ENSO). Unlike Fourier transforms, windowed Fourier transforms are used to analyse local frequency in a time series. However, they inadequately resolve low-frequency signals and also require analysis of numerous window lengths to determine the most suitable window, which is not appropriate if there are multiple dominant frequencies in the time series [4]. The wavelet transform efficiently resolves both high- and low-frequency signals by using local base functions which are stretched and translated in both frequency and time. This study uses the Morlet wavelet, which is described 
by

$$
\psi_{0}(\eta)=\pi^{-1 / 4} e^{i \omega_{0} \eta} e^{-\eta^{2} / 2},
$$

where $\eta$ is a non-dimensional time parameter, and $\omega_{0}$ the non-dimensional frequency. Given a time series $x_{n}$, the continuous wavelet transform is defined as the convolution

$$
W_{n}(s)=\sum_{n^{\prime}=0}^{N-1} x_{n^{\prime}} \psi^{*}\left(\frac{\left(n^{\prime}-n\right) \delta t}{s}\right),
$$

where $\mathrm{s}$ is the wavelet scale, $\mathrm{n}$ is a localised time index, $\mathrm{N}$ is the number of points in the time series and $*$ denotes the complex conjugate. In this study, the wavelet transform is computed in Fourier space as it is more efficient than directly computing (2). The discrete Fourier transform of $x_{n}$ is

$$
\widehat{\chi}_{k}=\frac{1}{N} \sum_{n=0}^{N-1} x_{n} e^{-2 \pi i k n / N},
$$

where $k=0,1, \ldots, N-1$ is the frequency index, and the Fourier transform of the function $\psi(t / s)$ is given by $\hat{\psi}(s \omega)$. Finally, by the convolution theorem and taking the inverse Fourier transform, the wavelet transform is

$$
W_{n}(s)=\sum_{k=0}^{N-1} \hat{x}_{k} \hat{\psi}^{*}\left(s \omega_{k}\right) e^{i \omega_{k} n \delta t}
$$

where

$$
\omega_{k}=\left\{\begin{array}{cl}
2 \pi k / N \delta t & \text { for } k \leqslant N / 2, \\
-2 \pi k / N \delta t & \text { for } k>N / 2 .
\end{array}\right.
$$

Equation (4) allows calculation of the continuous wavelet transform for a given wavelet scale using a routine such as the fast Fourier transform in MATLAB. The wavelet power spectrum is defined as

$$
\mathrm{P}(\mathrm{s})=\left|\mathrm{W}_{\mathfrak{n}}(\mathrm{s})\right|^{2}
$$


and is used to visualise the time evolution of periodic signals in a time series. In contrast, the global power spectrum is equivalent to applying a Fourier transform to the time series and thereby provides a global view of the dominant amplitudes. The global power spectrum is the average over local wavelet spectra through time

$$
\bar{W}^{2}(s)=\frac{1}{N} \sum_{n=0}^{N-1}\left|W_{n}(s)\right|^{2} .
$$

As the time series covers only a period of approximately 120 years, the wavelet analysis conducted only considers periods from 0-32 years.

\section{Results}

\subsection{Evolution of precipitation}

Figure 2 presents time series of precipitation for both Newcastle and Scone's respective wet season, and box plots of the bootstrapped mean wet season precipitation over 20-year periods. While having a consistent range on the vertical axes is ideal, more information is gleaned by not adjusting the range on the plots as wet season precipitation is completely different at each location.

Over the past century, wet season precipitation in Newcastle has remained steady, as seen by the lack of statistically significant differences between any two 20-year periods tested (Table 1). The frequency of years above the 90th percentile or below the 10th percentile has decreased, as have the tail lengths in the box plots, suggesting reduced variability in wet season precipitation for Newcastle (Figure 2). Scone's recorded wet season rainfall contrasts with Newcastle's, with a marked increase for the period 1938-1977, compared to the previous forty years. The mean wet season rainfall has since decreased to be consistent with amounts recorded in the early twentieth century. While the most recent two 20-year periods are similar (p-value 

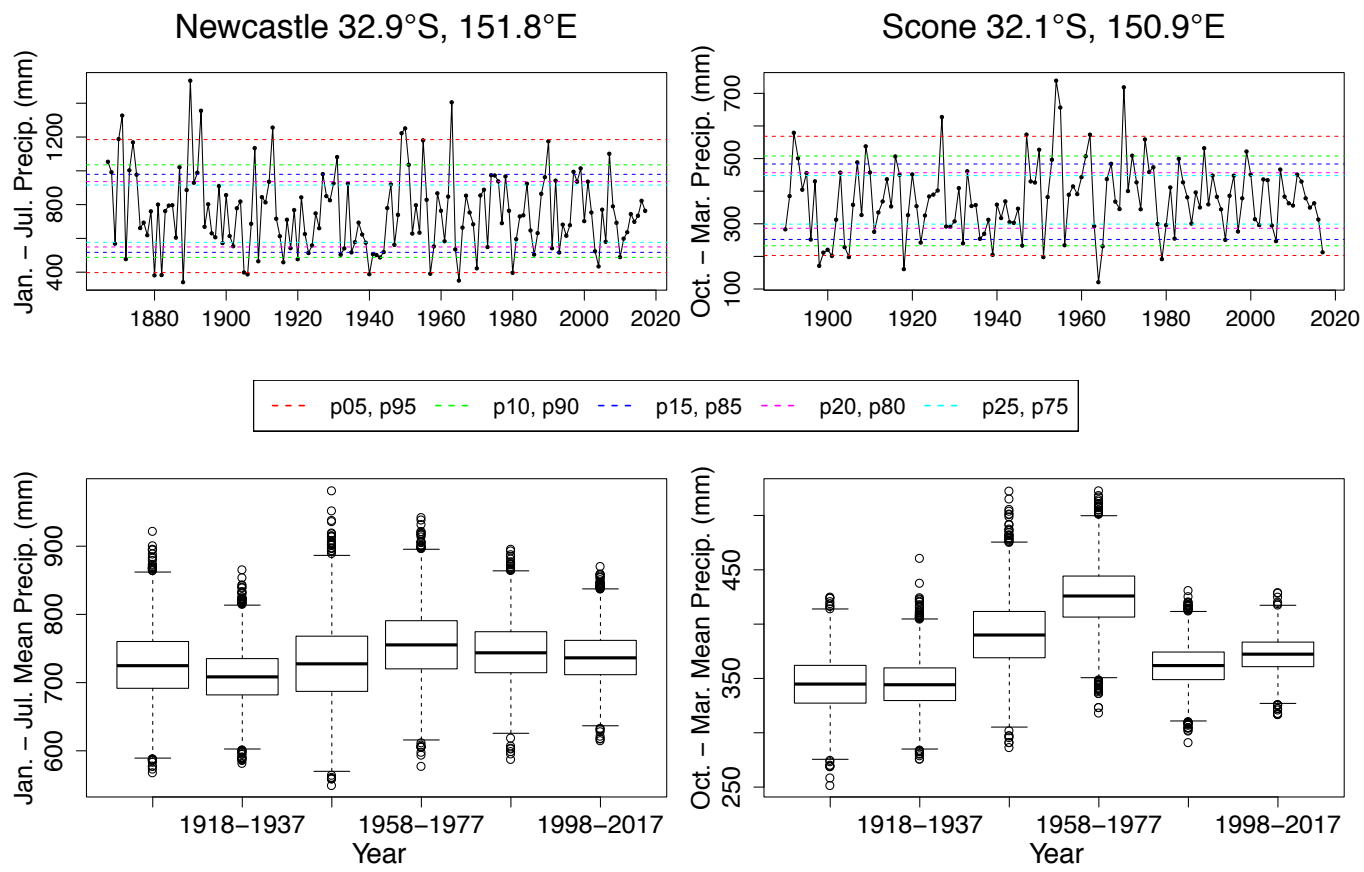

Figure 2: Time series of total wet season precipitation (top) and box plots of the bootstrapped mean wet season precipitation over 20-year periods (bottom) for Newcastle (left) and Scone (right). Dashed lines indicate the 5th and 95th (bottom and top red), 10th and 90th (bottom and top green) 15th and 85th (bottom and top dark blue), 20th and 80th (bottom and top pink), and 25th and 75th percentiles (bottom and top light blue). 
Table 1: The p-values for the permutation test on the difference between the mean wet season precipitation between two 20-year periods. Tests conducted used 5000 resamples.

\begin{tabular}{c|cc} 
Periods Compared & Newcastle & Scone \\
\hline $1958-1977$ v 1978-1997 & 0.840 & 0.072 \\
$1958-1977$ v 1998-2017 & 0.793 & 0.123 \\
$1978-1997$ v 1998-2017 & 0.900 & 0.694
\end{tabular}

$=0.694$, Table 1), the difference in precipitation between 1958-1977 and $1998-2017$ is statistically significant (p-value $=0.123$, Table 1$)$. Over the past 30 years, there has been a clear decrease in the frequency of years with recorded precipitation above the 85th percentile for Scone (Figure 2), a trend requiring continued monitoring.

\subsection{Evolution of temperature}

Figure 3 is the same as Figure 2, but for the mean maximum temperature (TMax) for each location's wet season. Similarly, Figure 4 provides an overview of the mean minimum temperature (TMin) for each location's wet season. If the trends in TMax and TMin are consistent, then the mean temperature should also remain roughly consistent with these trends.

Since the 1960s, a strongly increasing trend in TMax has been present in Newcastle. The last year with a record below the 5th percentile was during the 1960s, and since the mid-1980s there have been no mean maximum temperatures below the 25th percentile. The difference in TMax between the periods 1958-1977 and 1998-2017 is statistically significant (p-value $=0.000$, Table 2). Scone's TMax shows a rather interesting pattern. There was a decrease during 1938-1977, which is most likely due to increased cloud cover consistent with the increased precipitation during this period (Figure 2) and since the 1980s, the frequency of years recording TMax below the 10th percentile has increased. Despite this, the box plots for Scone show 

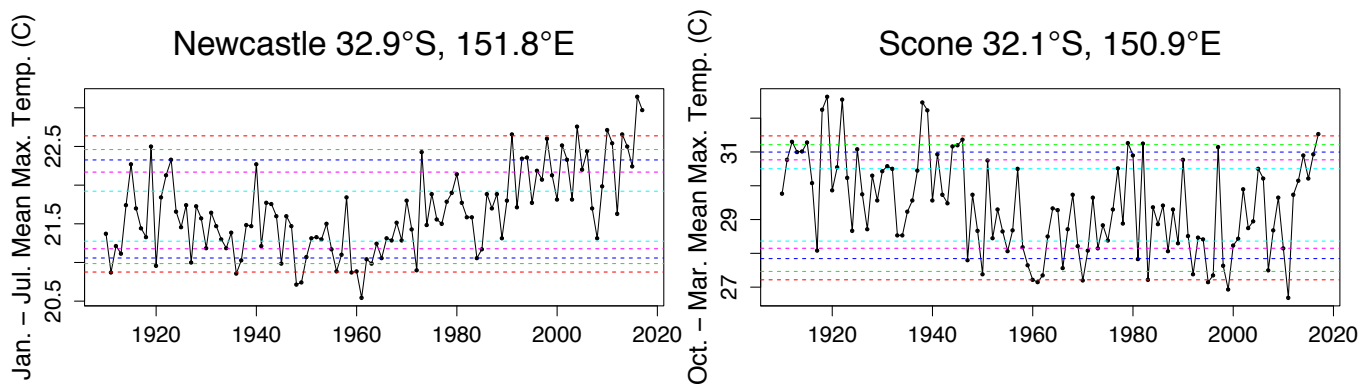

\begin{tabular}{|lllllllll}
\hline$\ldots$ & p05, p95 $\ldots$ & p 10, p90 & $\ldots$ & p15, p85 & $\ldots$ & p20, p80 & $\ldots$ & p25, p75 \\
\hline
\end{tabular}
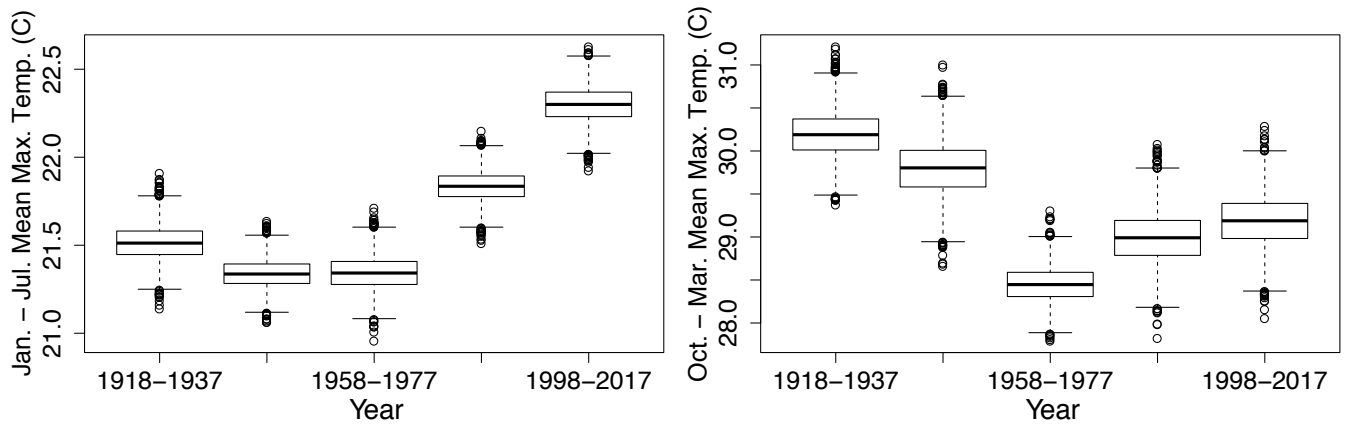

Figure 3: As in Figure 2, but for TMax during each locations' respective wet season.

an increasing trend emerging for TMax (Figure 3), with the difference in mean TMax between 1958-1977 and 1998-2017 being statistically significant $(\mathrm{p}$-value $=0.058$, Table 2$)$.

The TMin for Newcastle appears to begin increasing in the early 1980s, which is slightly later than TMax. This is evident through the increasing frequency of years with TMin recorded above the 90th percentile, particularly from the 1990s, and the decrease in frequency of years with TMin recorded below the 10th percentile from the 1960s (Figure 4). Further, there is a statistically significant difference between mean TMin in 1958-1977 and 1998-2017 (p-value $=0.000$, Table 2). These results, along with those for TMax, suggest that the mean wet season temperature for Newcastle has been increasing since at least 

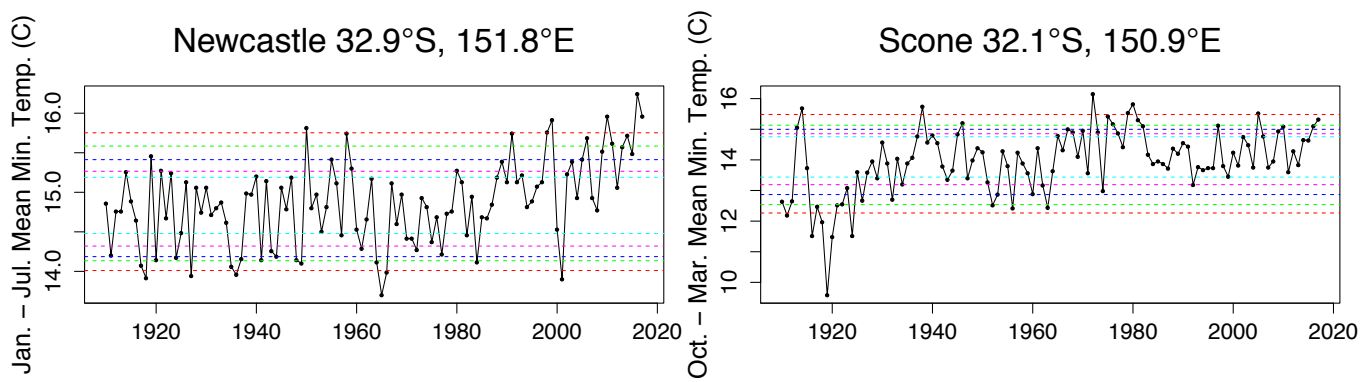

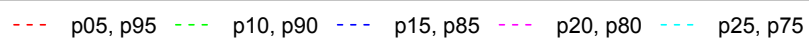
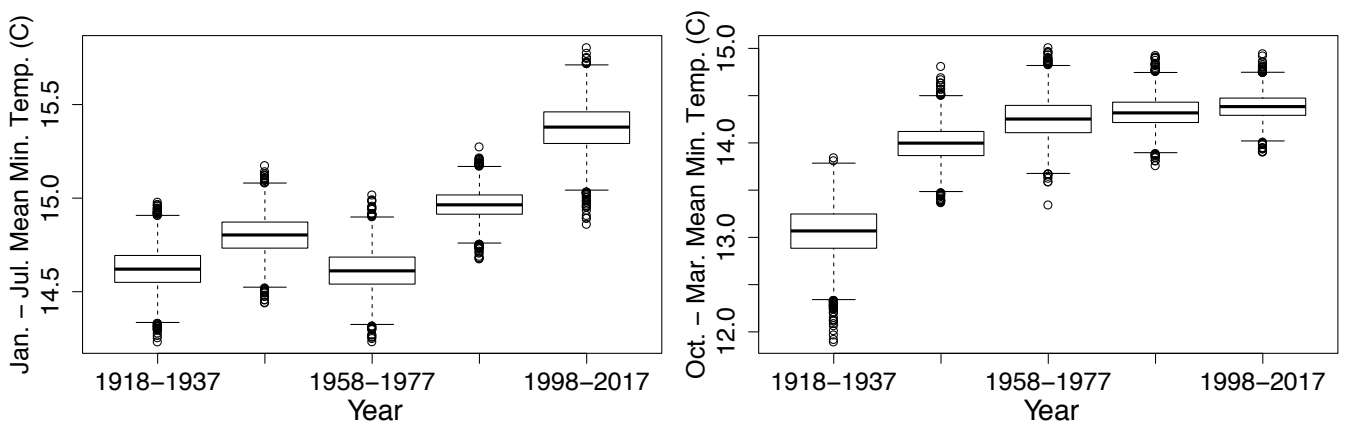

Figure 4: As in Figure 2, but for TMin during each locations' respective wet season.

Table 2: As in Table 1, but for the difference in the mean maximum temperature (TMax) and for the mean minimum temperature (TMin) during the wet season.

\begin{tabular}{c|cc|cc} 
& \multicolumn{2}{|c}{ TMax } & \multicolumn{2}{c}{ TMin } \\
Periods Compared & Newcastle & Scone & Newcastle & Scone \\
\hline $1958-1977$ v 1978-1997 & 0.001 & 0.162 & 0.015 & 0.787 \\
$1958-1977$ v 1998-2017 & 0.000 & 0.058 & 0.000 & 0.595 \\
$1978-1997$ v 1998-2017 & 0.002 & 0.661 & 0.007 & 0.783
\end{tabular}


the early 1980s. The TMin trends for Scone again show a different pattern. There is a clear increase in TMin between the years 1918-1937 and 1938-1957. However, TMin has since remained relatively constant. For example, there is no statistically significant difference between the years 1958-1977 and 1998-2017 ( $\mathrm{p}$-value $=0.595$, Table 2). While TMin is remaining steady, TMax for Scone has been increasing since the late 1970s, and so the mean wet season temperature for Scone may still be increasing overall.

\subsection{Wavelet analysis of precipitation}

Figure 5 shows the wavelet power spectra (6) and global power spectra (7) for wet season precipitation. Both locations show a clear two to seven year periodic signal, suggestive of an influence from the El-Niño Southern Oscillation (ENSO). The global power spectrum for Newcastle has one statistically significant peak around four years, while Scone has two peaks around both four and seven years. The seven year peak for Scone is clearly influenced by the high local power between 1940 and 1970 corresponding to the increase in precipitation occurring in this period, while the four year peak is related to a consistently appearing local signal. There is also a 16-32-year periodic signal which, while not statistically significant, appears to have some influence on the recorded precipitation for both sites.

\subsection{Wavelet analysis of temperature}

Wavelet spectra were also computed for wet season TMax and TMin (Figure 6). The TMax power spectra for both locations shows some power in the two to four year range, suggesting that ENSO influences TMax for both of these locations. From the 1970s, the number of years with high power in the two to four year range suggests that ENSO is more influential since then. There is high power in the two to seven year range for Newcastle's TMin, suggesting an ENSO influence. In contrast, Scone's TMin wavelet power spectra has little 

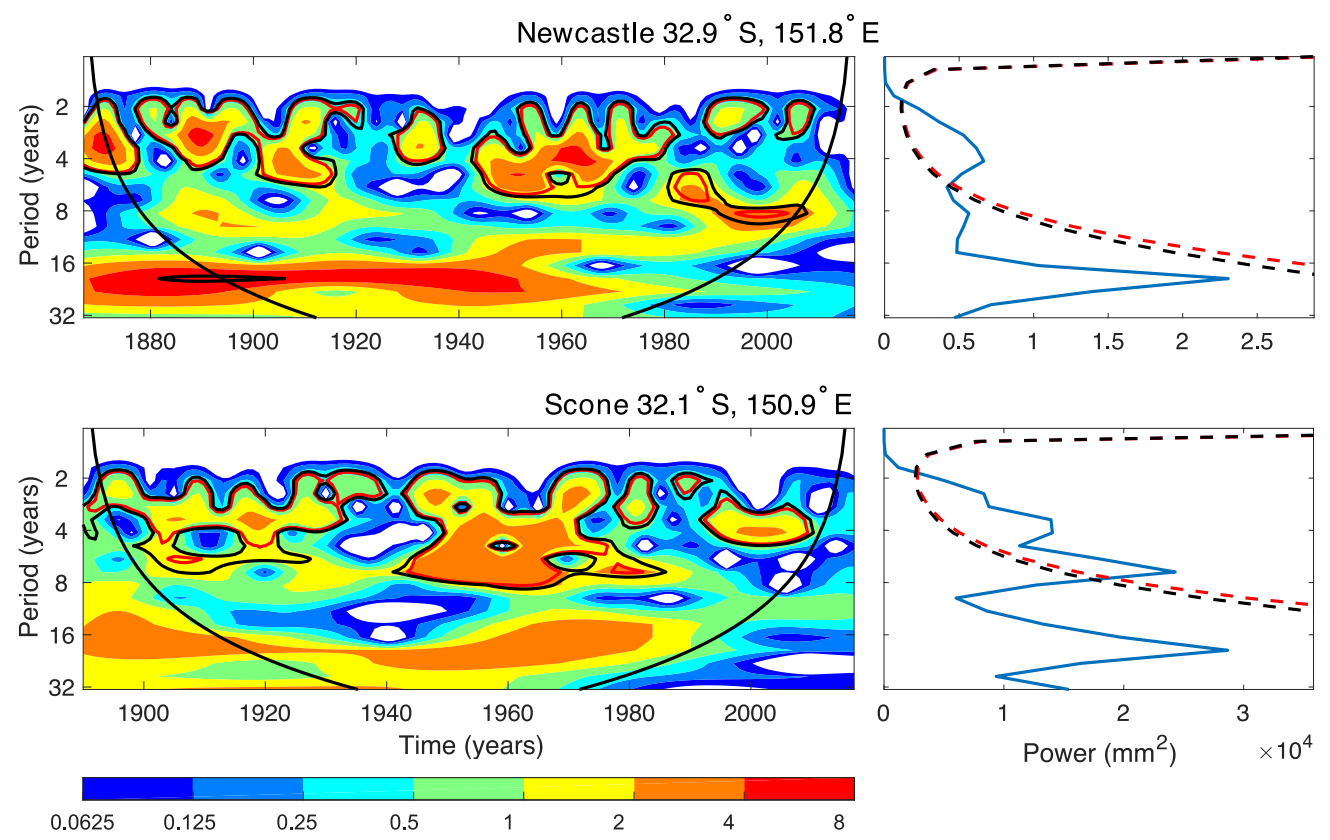

Figure 5: Wavelet analysis for total wet season precipitation for Newcastle and Scone. In the wavelet power spectrum (left), low values (blue) indicate low variability whereas high values (red) indicate high variability. Peaks in the global power spectrum (right) indicate high variability. The dashed/solid red line indicates the $95 \%$ confidence level, whereas the dashed/solid black line indicates the $90 \%$ confidence level. 


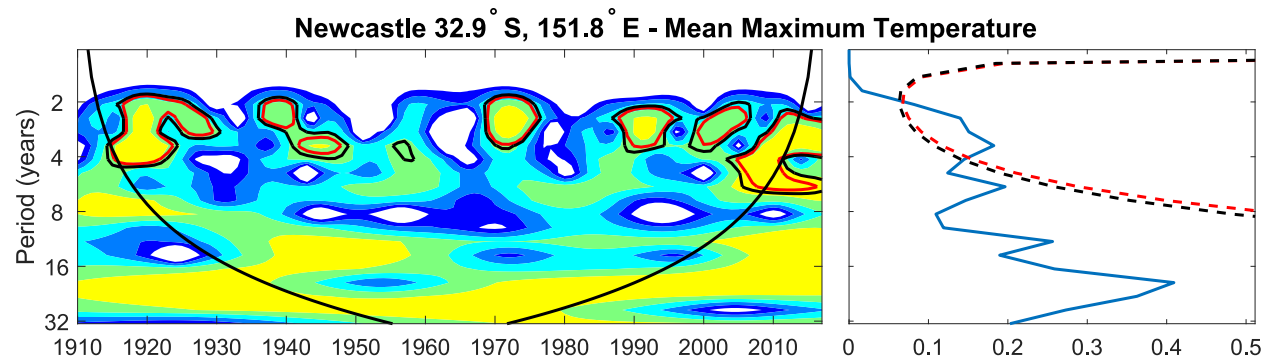

Scone $32.1^{\circ} \mathrm{S}, 150.9^{\circ} \mathrm{E}$ - Mean Maximum Temperature

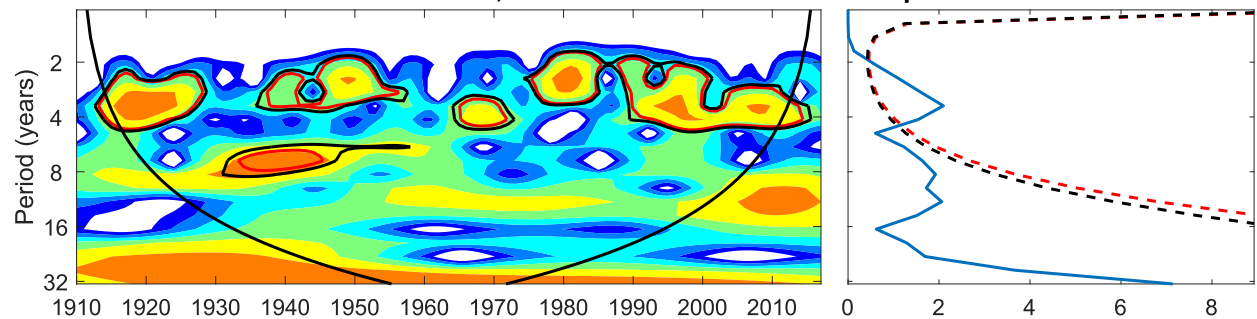

Newcastle $32.9^{\circ} \mathrm{S}, 151.8^{\circ} \mathrm{E}$ - Mean Minimum Temperature

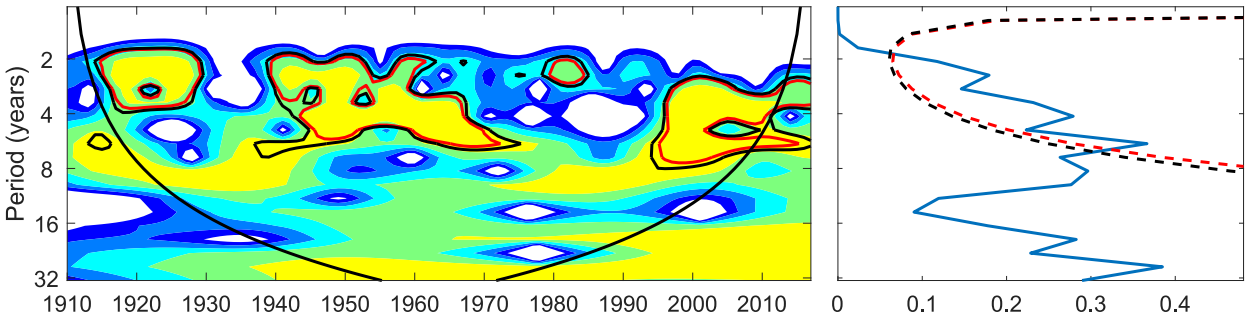

Scone $32.1^{\circ} \mathrm{S}, 150.9^{\circ} \mathrm{E}$ - Mean Minimum Temperature
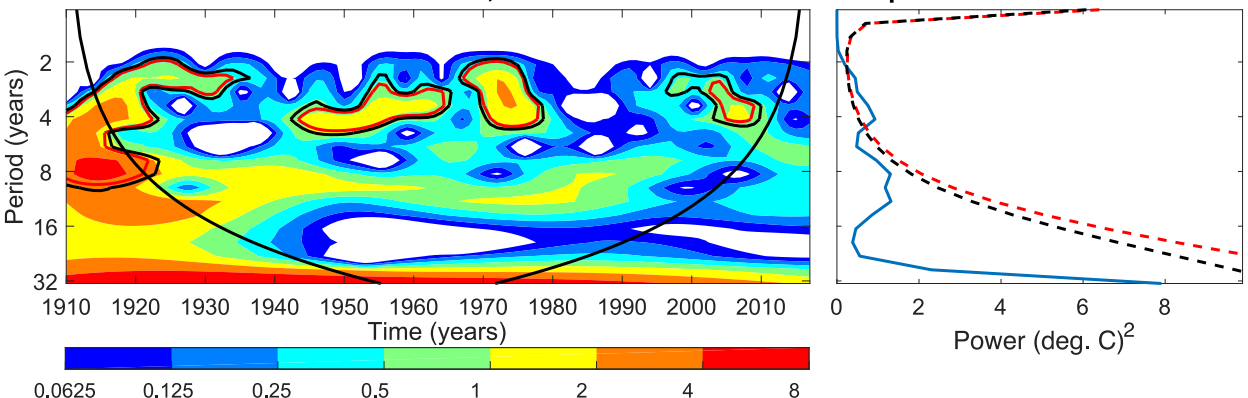

$\begin{array}{llllllll}0.0625 & 0.125 & 0.25 & 0.5 & 1 & 2 & 4 & 8\end{array}$

Figure 6: As in Figure 5, but for TMax (top two rows) and TMin (bottom two rows). 
power in the two to seven year range, except during the 1940s-1960s and the late 1960s-late 1970s. Consequently, Scone's global power spectra has no statistically significant peaks over any periods, implying little influence on Scone's TMin.

\section{Discussion and Conclusions}

Currently, intense drought is affecting much of southern and eastern Australia. This drought followed soon after the Millennium Drought, which affected southern Australia during 1997-2009. This study analysed wet season precipitation and temperature for a coastal and inland station, Newcastle and Scone, using statistical resampling methods and wavelets.

Newcastle precipitation was stable around its long term mean, though variability has decreased since the 1960s. In contrast, Scone's precipitation has fluctuated, with an increase during 1938-1977 followed by a marked decrease during 1978-2017. Wavelet analysis revealed a two to seven year signal influencing precipitation in both locations, suggesting some reliance on ENSO. A much longer (16-32-year) signal is also present in both time series. While a lack of precipitation is the main cause for drought, increasing temperatures increase evaporation and hence produce drier soils, thereby exacerbating the drought intensity such that previous rainfall deficits which may not have caused drought could now do so. Thus, an analysis of temperature trends is also necessary. In Newcastle, both the mean maximum temperature (TMax) and mean minimum temperature (TMin) have strongly increased since the mid-20th century. Scone's TMax has increased more gradually since this time, while TMin has hardly changed. This suggests that, overall, the mean temperature for both locations may be increasing, causing dry conditions to be harder felt. Wavelet analysis shows that ENSO may have an influence on TMax for both locations. Meanwhile, Newcastle's TMin is somewhat affected by ENSO, but a clear driver for TMin in Scone was not identified. 
Periods of dryness are clearly having greater impact, further accentuated by a growing population, increasing strain on water supplies. The current drought is drastically affecting the Hunter Valley, while coastal areas like Newcastle are relatively unscathed (Figure 1). Further study of meteorological factors causing this disparity, and of precipitation and temperature trends over eastern Australia is necessary to inform the agriculture and water management sectors. Without further detailed study of changes in precipitation and temperature, drought affected regions will be inadequately prepared, worsening the drought's effects.

Acknowledgements This research is supported by an Australian Government Research Training Program Scholarship and the ARC Centre of Excellence for Mathematical and Statistical Frontiers. We thank two anonymous reviewers for their suggestions which have improved the quality of the manuscript.

\section{References}

[1] Reisinger A., R. L. Kitching, F. Chiew, L. Hughes, P. C. D. Newton, S. S. Schuster, A. Tait, and P. Whetton. Climate change 2014: Impacts, adaptation, and vulnerability. Part B: Regional aspects. Contribution of Working Group II to the Fifth Assessment of the Intergovernmental Panel on Climate Change. Tech. rep. Intergovernmental Panel on Climate Change, 2014. Chap. 25, pp. 1371-1438. URL: https://www.ipcc.ch/report/ar5/wg2/ (cit. on p. C111).

[2] Bureau of Meteorology. Climate Glossary-Drought. URL: http://www.bom.gov.au/climate/glossary/drought.shtml (cit. on p. C110). 
[3] Bureau of Meteorology. Climate Maps-Rainfall Latest. URL: http: //www . bom.gov.au/jsp/awap/rain/index . jsp?colour=colour\& time=latest\&step=0\&map=decile\&period=24month\&area $=$ nat (cit. on p. C112).

[4] K. M. Lau and H. Weng. "Climate signal detection using wavelet transform: How to make a time series sing". In: B. Am. Meteorol. Soc. 76 (1995), pp. 2391-2402. DOI:

10.1175/1520-0477(1995)076<2391: CSDUWT>2.0.C0;2 (cit. on p. C113).

[5] M. B. Richman and L. M. Leslie. "The 2015-2017 Cape Town drought: Attribution and prediction using machine learning". In: Procedia Comput. Sci. 140 (2018), pp. 248-257. DOI:

10.1016/j.procs.2018.10.323 (cit. on p. C111).

[6] M. B. Richman and L. M. Leslie. "Uniqueness and causes of the California drought". In: Procedia Comput. Sci. 61 (2015), pp. 428-435. DOI: 10.1016/j.procs.2015.09.181 (cit. on p. C111).

[7] C. Torrence and G. P. Compo. "A practical guide to wavelet analysis". In: B. Am. Meteorol. Soc. 79 (1998), pp. 61-78. DOI:

10.1175/1520-0477(1998) 079<0061:APGTWA>2.0.CO;2 (cit. on p. C113).

\section{Author addresses}

1. J. Hartigan, School of Mathematical and Physical Sciences, University of Technology Sydney, Ultimo, New South Wales 2007, Australia.

mailto: joshua.hartigan@student.uts . edu . au orcid:0000-0002-7783-9440

2. S. MacNamara, School of Mathematical and Physical Sciences, University of Technology Sydney, Ultimo, New South Wales 2007, 
Australia.

3. L. M. Leslie, School of Mathematical and Physical Sciences, University of Technology Sydney, Ultimo, New South Wales 2007, Australia. 